\title{
Editorial
}

\section{Dengue vaccines: closer but not there yet}

As another high-transmission season approaches in Brazil, dengue virus (DENV) with its four serotypes, becomes a major subject in the news for both the general population and health authorities. DENV vaccines have been sought since the Second World War without much success, despite the numerous methods employed by several research groups worldwide. Much of the emphasis has been placed on the development of live attenuated viruses (LAVs) (Supplementary data). Based on the advancements made possible by recombinant DNA technology and the successful propagation of DENV in cells certified for the production of vaccines, in 1987, the World Health Organization (Brandt 1990) presented a work program that forecast the registration of a dengue vaccine within five years. This prediction proved to be overly optimistic, as the first clinical trials were not completed until a decade after the pronouncement.

The development of modern cell cultures, together with the capability of manipulating the flavivirus genome, has allowed the construction of recombinant LAVs, some of which reached clinical trials. Among these LAVs, a set of chimeric yellow fever 17D vaccine viruses bearing the envelope genes of each of the four DENV serotypes has progressed to Phase III clinical trials. Other genetically engineered chimeric DENV LAVs have been developed and are being tested for their safety and immunogenicity in man (Supplementary data). It is noteworthy that, until recently, the question remained whether there would ever be a licensed vaccine for widespread use. However, efforts by several laboratories have been successful and we are now met with the possibility of the registration of the first generation of DENV vaccines. Interestingly, these vaccines will likely be recombinant DNA-derived LAVs.

With respect to registration, Phase III clinical trials must include protracted follow-up of volunteers living in DENV-endemic areas. This requirement is because prior exposure may increase or decrease the effectiveness of the vaccine under evaluation and there is a theoretical risk that waning titres of neutralising antibody may facilitate severe disease following infection with a wild DENV - the clinical symptoms range from an asymptomatic infection to severe syndromes, dengue haemorrhagic fever and dengue shock syndrome. Waning antibody titres and their potential role in more severe dengue infections are the reason why long-lasting tetravalent seroconversion is considered an essential outcome of vaccination. With regard to the establishment of efficacy in these trials, the seasonal and geographical variabilities in the circulation of the individual strains of DENV necessitate in-depth evaluation to identify the sites that will be most likely to provide disease incidence data suitable for establishing vaccine efficacy.

The tetravalent LAV vaccine from Sanofi-Pasteur is in the most advanced stage of development and efficacy studies have already been initiated in different countries. Hopefully, this vaccine will be licensed in three years (2014), when it might be used in public vaccination campaigns. A study by Amarasinghe and Mahoney (2011) estimated the likely number of doses of dengue vaccine needed in Brazil under different scenarios in the first five years after the introduction of the vaccine. Countrywide, roughly 8-60 million doses would be needed for vaccination of the 12-23month cohort plus 130-400 million doses for catch-up vaccination of individuals up to 40 years of age (Amarasinghe \& Mahoney 2011). The question regarding the need for a booster dose remains open and would add another layer of complexity in predicting vaccine needs.

Given the demand and limited production capacity within at least the first five years after introduction, there will be a need to prioritise the age groups and localities that will receive the vaccination. The information needed to support these decisions is currently the main point of discussion of health authorities. Surveillance data specific to each country will be needed to guide the introduction of the vaccine, especially in the context of limited availability. Estimates of disease incidence and associated costs are based solely on the number of reported cases, which inevitably underestimates the magnitude of the problem. Efficient surveillance systems and seroprevalence studies are needed to provide more precise estimates of the incidence of the disease at both regional and national levels to support decision-making for the optimal use of the available vaccine.

However, it cannot be assumed that vaccination will solve the dengue problem. Vaccines will help, but the continuous control of this disease will also require the reduction of mosquito vector populations with environmental management. Even so, it will take time to reduce transmission rates (Gubler 2011).

As candidate vaccines reach critical milestones in their development, the main issue is no longer the feasibility of developing an effective vaccine against dengue but how to make best use of this vaccine in the five years following its introduction, during which there will be a high demand, low availability and yet undefined costs (Amarasinghe et al. 2010). 


\section{REFERENCES}

Amarasinghe A, Mahoney RT 2011. Estimating potential demand and supply of dengue vaccine in Brazil. Hum Vaccin 7: 776-780.

Amarasinghe A, Wichmann O, Margolis HS, Mahoney RT 2010.
Forecasting dengue vaccine demand in disease endemic and nonendemic countries. Hum Vaccin 6: 745-753.

Brandt WE 1990. Development of dengue and Japanese encephalitis vaccines. J Infect Dis 162: 577-583.

Gubler DJ 2011. Emerging vector-borne flavivirus diseases: are vaccines the solution? Expert Rev Vaccines 10: 563-565. 
Main approaches to dengue virus (DEN) vaccine development

\begin{tabular}{llll}
\hline Vaccine & Approach & Laboratory & Current status \\
\hline 1 & Live attenuated, PDK cells & Mahidol University/Sanofi-Pasteur & Up to Phase II/discontinued \\
2 & Live attenuated, FRhL and PDK cells & WRAIR/GSK & Up to Phase II/discontinued \\
3 & Live attenuated viral vectors & & Phase III \\
& 17D-based & Acambis/Sanofi-Pasteur & Pre-clinical \\
& & Fiocruz & Phase I \\
& DEN2 PDK53-based & Invitrogen & Pre-clinical \\
4 & Measles-based & Pasteur Institute & Pre-clinical \\
5 & Killed virus & WRAIR/GSK/Fiocruz & Phase I \\
6 & Subunit protein & Merck/Hawaii Biotech & Pre-clinical \\
\hline
\end{tabular}

FRhL: fetal rhesus monkey lung; PDK: primary dog kidney; WRAIR/GSK: Walter Reed Army Institute of Research/ GlaxoSmithKline. 